\title{
Application of Weakest Link Probabilistic Framework for Fatigue Notch Factor to Turbine Engine Materials
}

\author{
Oluwamayowa A. Okeyoyin", Gbadebo M. Owolabi \\ Department of Mechanical Engineering, Howard University, Washington DC, USA \\ Email: *oluwamayowa.okeyoy@bison.howard.edu
}

Received May 21, 2013; revised June 24, 2013; accepted July 23, 2013

Copyright (C) 2013 Oluwamayowa A. Okeyoyin, Gbadebo M. Owolabi. This is an open access article distributed under the Creative Commons Attribution License, which permits unrestricted use, distribution, and reproduction in any medium, provided the original work is properly cited.

\begin{abstract}
This paper is concerned with the extension of a recently developed probabilistic framework based on Weibull's weakest link and extreme-value statistics to aero-engine materials like titanium alloy and nickel-base super alloys using simulation strategies that capture both the essence of notch root stress gradient and the complexity of realistic microstructures. In this paper, notch size effects and notch root inelastic behavior are combined with probability distributions of microscale stress-strain gradient and small crack initiation to inform minimum life design methods. A new approach which can be applied using crystal plasticity finite element or closed-form solution is also proposed as a more robust approach for determining fatigue notch factor than the existing classical methods. The fatigue notch factors predicted using the new framework are in good agreements with experimental results obtained from literature for notched titanium alloy specimens subjected to uniaxial cyclic loads with various stress ratio.
\end{abstract}

Keywords: Microstructure; Fatigue; Crack Initiation; Crystal Plasticity

\section{Introduction}

Titanium alloy is widely used in aero engine components. The fatigue resistance of aero-engine components made from this material can be drastically reduced by the presence of small notches on the components formed from the ingestion of foreign objects causing foreign object damage (FOD) [1-2]. To account for the effects of FOD on the fatigue strength of these materials, the damage are usually modeled as notches with a certain depth and notch root radius [1-2]. The severity of these notches in materials is characterized by the elastic stress concentration factor, $k_{t}$ which is the ratio of peak (maximum) local stress at the notch root to the remotely applied stress, $S$ as shown in Figure 1.

$$
k_{t}=\frac{\sigma_{\text {peak }}}{S}
$$

$k_{t}$ is dimensionless and a compilation of its values for different notch geometries and loading modes can be found in Peterson's book [3]. However, $k_{t}$ under-estimates fatigue life and several arguments have been attributed to this observation. The fatigue life of notched

${ }^{*}$ Corresponding author. component is not only dependent on the peak stress as predicted by $k_{t}$, but also on the average stress that acts over a finite damage process zone. Consequently, fatigue life prediction methods based on $k_{t}$ typically do not consider stress gradients which have been shown to influence the fatigue life of complex notched components [4-5]. Thus, the actual reduction factor on long fatigue lives is typically represented by the concept of fatigue notch factor, otherwise known as the fatigue strength reduction factor, $k_{f}$.

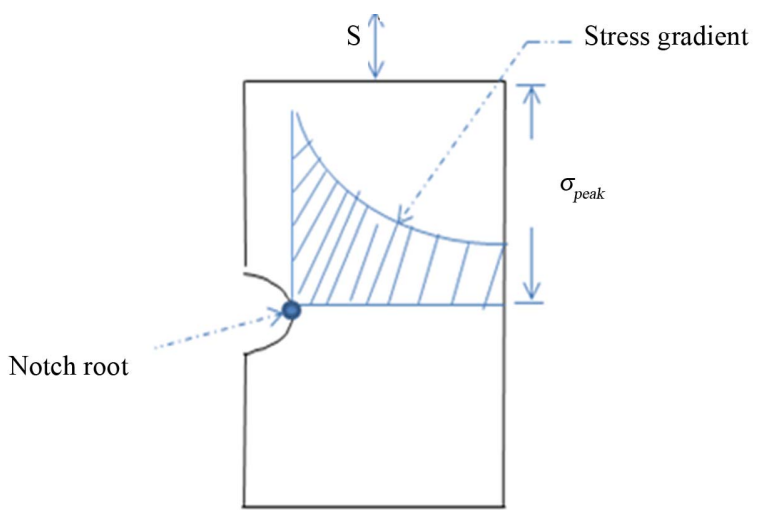

Figure 1. Stress distributions in a notched specimen. 
The fatigue notch factor, $k_{f}$, is determined at a given number of completely reversed cycles (typically $10^{6}$ or $10^{7}$ ) to crack initiation and is given as:

$$
k_{f}=\frac{\sigma_{f}^{\text {unnotched }}}{\sigma_{f}^{\text {notched }}}
$$

where $\sigma_{f}^{\text {unnotched }}$ is the fatigue strength of the unnotched specimen and $\sigma_{f}^{\text {notched }}$ is the fatigue strength of the notched specimen. The difference between $k_{t}$ and $k_{f}$ for a given microstructure is typically represented by the notch sensitivity factor, $q$ given as:

$$
q=\frac{k_{f}-1}{k_{t}-1}
$$

At $q=0$, there is no notch sensitivity and at $q=1$ we have full notch sensitivity, i.e., full theoretical elastic concentration effect. Several empirical relations have been developed to estimate the fatigue notch factor of a material and its associated notch sensitivity index. These techniques include: the classical methods (Neubers [6-7], Peterson [3,8] and Heywood [9]), stress field intensity method [10], and probabilistic method based on linear elastic fracture mechanics [11]. Detail review of these methods can be found in [12], each attempt to simplify the complex behavior of fatigue in notched components to a few geometric and characteristic material constants. However, these approaches suffer from some fundamental drawbacks. Among these drawbacks is that the fatigue notch factors are obtained through time consuming and costly experiments. Moreover, the relationship of microstructure to $K_{f}$, using these constants has proven difficult to establish. Recently, Owolabi et al. [14] have established a probabilistic framework based on weakest link theory and extreme-value statistics which incorporates information regarding the peak stress and stress gradient relative to microstructure length scales within a well defined fatigue damage process zone around the notch root. This paper combines the developed probabilistic framework with other existing probabilistic formulations that consider the size distribution and different competing damage mechanisms for aero-engine materials.

\section{Material Systems}

The alloy used for this study is a dual-phase titanium alloy, Ti-6Al-4V. Ti-Al alloys offer a range of properties such as high strength and fracture toughness at low temperatures to high strength and creep resistance at elevated temperatures. These wide ranges of properties have led to extensive use of Ti-Al alloys in engineering applications from airframe components to compressor blades applications. The experimental data used in this work were obtained from Haritos et al. [1], Lanning et al. [13] and Naik et al. [15] on Ti-6Al-4V for various notch root ge- ometries and stress ratios. The Ti-6Al-4V specimens used in these papers were obtained from forged bar, which were initially heat treated to $705^{\circ} \mathrm{C}$ for $2 \mathrm{~h}$, and then followed by static argon cooling to below $149^{\circ} \mathrm{C}$. The material was subsequently annealed in vacuum at $549^{\circ} \mathrm{C}$ for $2 \mathrm{~h}$. This is then followed by static argon cooling to below $149^{\circ} \mathrm{C}$. The microstructure of the resulting bar is as shown Figure 2.

\section{Crystal Plasticity Model of Ti-6Al-4V}

Crystal plasticity models are more suited for studying heterogeneity and interaction across grains in the notch root field as they relate grain scale stress to crystallographic slip response [16-18]. The use of crystal plasticity is thus relevant for the accurate prediction of the stress-strain field response at the notch root. The crystal plasticity model used in this work is based on 3D crystal plasticity models developed by Mayeur and McDowell (2007) [19]. Thus, only the summary of the crystal plasticity constitutive models is presented in this section.

The deformation in the material is based on a standard two term multiplicative decomposition of the deformation gradient into elastic and plastic parts, i.e.,

$$
F=F^{e} F^{p}
$$

Here, $F$ is the total deformation gradient, $F^{p}$ captures the dislocation glide through the lattice while $F^{e}$ captures the rigid body rotation and elastic stretching of the lattice. The plastic velocity gradient $\hat{L}^{p}$ defined as the sum of the crystalline shear displacement rates over all slip systems $k$ is given in the isoclinic, lattice invariant intermediate configuration as [20]:

$$
\hat{L}^{p}=\dot{F}^{p} \cdot\left(F^{p}\right)^{-1}=\sum_{k=1}^{N_{s y s}} \dot{\gamma}^{k}\left(S_{0}^{k} \otimes n_{0}^{k}\right)
$$

Here, $\gamma^{k}$ is the slip system shearing rate, and $S_{0}^{k}$ and $n_{0}^{k}$ are fixed unit vectors in the slip direction and slip plane normal direction, respectively. The slip vectors $S_{0}^{k}$ and $n_{0}^{k}$ remain unchanged through deformation $F^{p}$

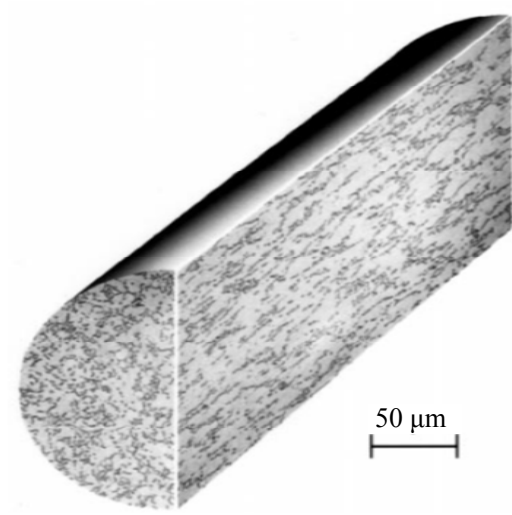

Figure 2. Ti-6Al-4V forged plate microstructure [1]. 
from the reference to the intermediate configuration and maintain orthogonality through $F^{e}$. The relationship between the slip system shearing rate and the resolved shear stress of the $k_{t h}$ slip system is described by the power law flow rule given by McGinty [21] as:

$$
\dot{\gamma}^{k}=\dot{\gamma}_{0}\left\langle\frac{\left|\tau^{k}-\chi^{k}\right|-\kappa^{k}}{D^{k}}\right\rangle^{M} \operatorname{sgn}\left(\tau^{k}-\chi^{k}\right)
$$

Here, $\gamma_{0}$ is the reference shearing rate, $\mathrm{M}$ is the inverse strain-rate sensitivity exponent which controls the rate sensitivity of flow, $\tau^{k}$ is the resolved shear stress, $\chi^{k}$ is the back stress, $\kappa^{k}$ is the length scale-dependent threshold stress and $D^{k}$ is the drag stress. As developed by Zhang et al. [22], the drag stress is taken as a non-evolving constant, while the back stress evolves according to an Armstrong-Frederick direct hardening/dynamic recovery type of equation, i.e.,

$$
\dot{\chi}^{k}=h \dot{\gamma}^{k}-h_{D} \chi^{k}\left|\dot{\gamma}^{k}\right|
$$

With $\chi^{k}(0)=0$. The threshold stress is expressed as

$$
\kappa^{k}=\frac{\kappa_{y}}{\sqrt{d^{k}}}+\kappa_{s}^{k}
$$

\section{Simulation of Notched Components}

The crystal plasticity constitutive model was coded into ABAQUS 2006 UMAT, based on previous work by Zhang et al. [22]; Mayeur and McDowell, 2007 [19]. For textured Ti-6Al-4V alloy, some of the material parameters in the crystal plasticity are obtained from Bridier et al. [23]. Finite element simulation was performed on three different geometries, meshed using 3D stress fournode linear tetrahedron element type (C3D4) and consisting of approximately 218940 elements to estimate the stress distribution and possible plastic straining that occur in the notched specimens. The dimensions of the specimens used and the different test cases are as given in Table 1. A diagram of the gage section of the specimen is provided in Figure 3.

Table 1. The 7 different test cases.

\begin{tabular}{cccccc}
\hline $\begin{array}{c}\text { Test } \\
\text { Case }\end{array}$ & $K_{t}$ & $\begin{array}{c}\text { Notch radius, } \\
\rho(\mathrm{mm})\end{array}$ & $\begin{array}{c}\text { Notch } \\
\text { depth, } \\
\mathrm{h}(\mathrm{mm})\end{array}$ & R-ratio & $\begin{array}{c}\text { Average alternating } \\
\text { HCF strength at } 10^{6} \\
\text { cycles (MPa) }\end{array}$ \\
\hline 1 & 2.78 & 0.330 & 0.729 & -1 & 173.6 \\
2 & 2.78 & 0.330 & 0.729 & 0.1 & 158.9 \\
3 & 2.78 & 0.330 & 0.729 & 0.5 & 104.6 \\
4 & 2.78 & 0.203 & 0.254 & 0.10 & 167.2 \\
5 & 2.78 & 0.203 & 0.254 & 0.50 & 105.2 \\
6 & 2.78 & 0.127 & 0.127 & 0.10 & 144.7 \\
7 & 2.78 & 0.127 & 0.127 & 0.50 & 111.0 \\
\hline
\end{tabular}

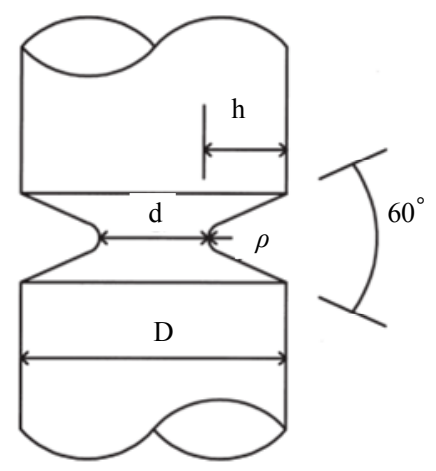

Figure 3. Gage section of the cylindrical specimen with a circumferential V-notch [1].

To reduce computational time, the notched specimen geometries are decomposed into three different regions: an outermost region, far from the notch root, where isotropic linear elasticity is used; an intermediate transition region where macroscopic $J_{2}$ cyclic plasticity theory is used; and finally the notch root region where crystal plasticity theory is used. The element size at the crystal plasticity region was chosen to coincide with the average grain size of Ti-6Al-4V which is $45 \mu \mathrm{m}$. The domain decomposition is as shown in Figure 4. Also, one quarter of the cylindrical notched specimen was modeled because of the symmetry in loading and geometry of the specimen as shown in Figure 5.

The bottom of the notched specimen is encastre while symmetry boundary conditions are applied to the two planes of symmetry. The notched specimens were tested at four different load ratios; $\mathrm{R}=0.1, \mathrm{R}=0.5$ and $\mathrm{R}=-1$. Average alternating HCF strength at $10^{6}$ cycles, as determined by Naik et al. [15] and as contained in Table 1 for different load ratios, are applied to the top of the specimen.

This paper is concerned with the extension of a recently developed probabilistic framework based on Weibull's weakest link and extreme-value statistics to aero-engine materials like titanium alloy and nickel-base super alloys using simulation strategies that capture both the essence of notch root stress gradient and the complexity of realistic microstructures. In this paper, notch size effects and notch root inelastic behavior are combined with probability distributions of microscale stress-strain gradient and small crack initiation to inform minimum life design methods. A new approach which can be applied using crystal plasticity finite element or closed-form solution is also proposed as a more robust approach for determining fatigue notch factor than the existing classical methods. The fatigue notch factors predicted using the new framework are in good agreements with experimental results obtained from literature for notched titanium alloy specimens subjected to uniaxial cyclic loads with various stress ratio. 


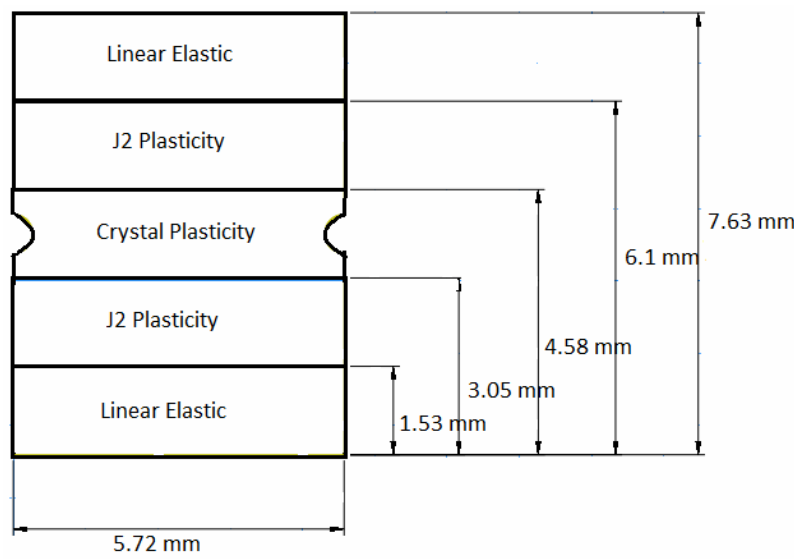

Figure 1. Domain decomposition of the cylindrical notched specimen geometry.

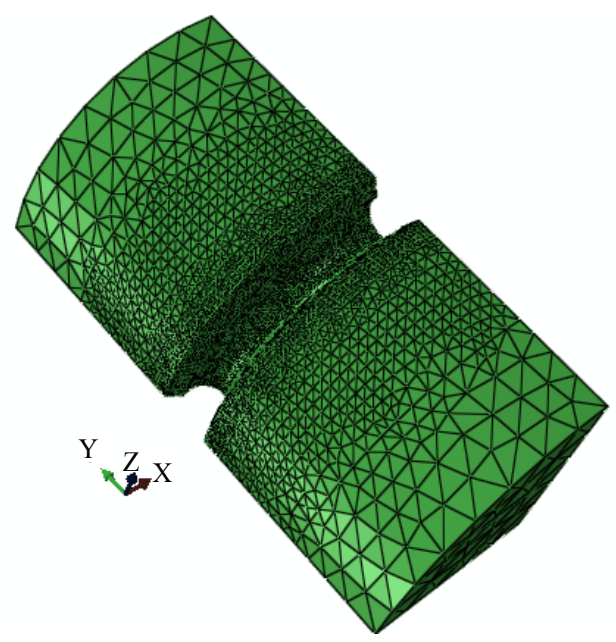

Figure 2. Finite element mesh for $0.33 \mathrm{~mm}$ notch root radius and $K_{t}=2.78$ consisting of four-node linear tetrahedron element type (C3D4).

For a smooth specimen with defects having a fatigue damage process zone of volume $V$, the whole volume is divided into small volume elements, $\mathrm{d} V$ with probability of failure of a sufficiently small volume element given as:

$$
\mathrm{d} P=\lambda \mathrm{d} V
$$

where here, $\lambda$ is the critical defect density defined as the expected number of defects per unit volume of the smooth specimen. Using weakest link theory, the probability of survival of the entire volume is obtained from the probability of survival of all " $m$ " number of subvolumes i.e.

$$
P_{s}=\prod_{i=1}^{m}\left(1-\mathrm{d} P_{i}\right)=\prod_{i=1}^{m}(1-\lambda \mathrm{d} V)
$$

This equations assumes that the defects are randomly distributed within the volume and thus do not interact, which is only reasonable when considering the formation of a fatigue crack(s) in high cycle (HCF) and very high cycle fatigue (VHCF) regimes. Following the framework presented in [14], as the volume of each small element tends to zero, Equation (10) can be transformed into

$$
P_{s}=\exp \left(-\int_{V_{d}} \lambda \mathrm{d} V\right)
$$

Using the generalized extreme value distribution function, the distribution of defects, $a$, that are above the threshold, $a_{t h}$, is modeled by a power law of the form

$$
\lambda=\frac{1}{V_{0}}\left(1+\xi\left(\frac{a-a_{t h}}{a_{0}}\right)\right)^{-1 / \varepsilon}
$$

where $a_{0}$ and $\xi$ are the scale and shape parameters respectively. Substituting (12) into (11) yields

$$
P_{s}=\exp \left\{-\frac{1}{V_{0}} \int_{V_{d}}\left(1+\xi\left(\frac{a-a_{t h}}{a_{0}}\right)\right)^{-1 / \varepsilon} \mathrm{d} V\right\}
$$

If $a_{t h}=a_{0}^{*} \xi$, re-arranging Equation (13) yields,

$$
P_{s}=\exp \left\{-\frac{1}{V_{0}} \int\left(\frac{a}{a_{0}^{*}}\right)^{-1 / \varepsilon} \mathrm{d} V\right\}
$$

where $a_{0}^{*}$ is regarded here as the mean defect size. Equation (14) is valid only if $\xi \neq 0$. The critical defect size is related to the microscopic stress (taking here as a random variable) through a power law relationship of the form

$$
\sigma=\frac{A}{\sqrt[z]{a}}
$$

where $\mathrm{A}$ and $\mathrm{z}$ are materials constants. Similarly, the stress amplitude, $\sigma_{0}$ corresponding to the mean defect size $a_{0}^{*}$ can be taken as the fatigue limit of the reference volume $V_{\mathrm{o}}$ for $50 \%$ failure probability. The two parameters can also be related by a power law of the form:

$$
\sigma_{0}=\frac{A}{\sqrt[z]{a_{0}^{*}}}
$$

Combining Equations (15) and (16) we have

$$
\frac{a}{a_{0}^{*}}=\left(\frac{\sigma_{0}}{\sigma_{a}}\right)^{z}
$$

Substituting Equation (17) into Equation (14) yields

$$
P_{s}=\exp \left\{-\frac{1}{V_{0}} \int\left(\frac{\sigma}{\sigma_{0}}\right)^{b} \mathrm{~d} V\right\}
$$

where $b=z / \xi$. For $\xi>0$, b and $\sigma_{0}$ represents a 2-parameter Weibull shape and scale parameters. The cumulative probability of HCF failure of the component, specifically defined can be obtained from Equation (18) as 


$$
P_{f}=1-\exp \left\{-\frac{1}{V_{0}} \int\left(\frac{\sigma}{\sigma_{0}}\right)^{b} \mathrm{~d} V\right\}
$$

To facilitate development of the expression for fatigue notch factor from Equation (18), the concept of stress homogeneity factor that have been used is introduced here. Thus Equation (18) can be re-written as,

$$
P_{f}=1-\exp \left\{-\frac{k V}{V_{o}}\left(\frac{\sigma_{\max }}{\sigma_{0}}\right)^{b}\right\}
$$

where

$$
k=\frac{1}{V} \int\left(\frac{\sigma}{\sigma_{\max }}\right)^{b} \mathrm{~d} V
$$

is regarded as the stress homogeneity factor. Conventionally, the fatigue notch factor is the ratio of unnotched to notched fatigue strength at the same probability of failure (usually 50\%). Using Equation (20), the probability of failure of unnotched specimen and a notched specimen will be the same when

$$
\exp \left\{-\frac{k_{s} V_{s}}{V_{o}}\left(\frac{\sigma_{\max , s}}{\sigma_{0}}\right)^{b}\right\}=\exp \left\{-\frac{k_{n} V_{n}}{V_{o}}\left(\frac{\sigma_{\max , n}}{\sigma_{0}}\right)^{b}\right\}
$$

where the subscripts $n$ and $s$ represent the respective value of the variable for notched and smooth (unnotched) specimens. The ratio of the smooth to notch fatigue driving force parameters (i.e., the stress amplitude) is used to define a new fatigue notch factor given as

$$
k_{f}=\frac{\sigma_{\max , s}}{\sigma_{\max , n}}=\left(\frac{k_{n}}{k_{s}}\right)^{1 / b}\left(\frac{V_{n}}{V_{s}}\right)^{1 / b}
$$

For smooth specimen that is loaded at a very low stress or strain amplitude in the HCF regime, the number of critically stressed grains (or elements) is very small. Thus for the life limiting case in which only one grain or element is critically stressed above the threshold, $V_{s}=V_{e}$ (i.e., volume of element or grain) and $K_{s}=1$; thus Equation (23) becomes

$$
\begin{aligned}
k_{f} & =\frac{\sigma_{\max , s}}{\sigma_{\max , n}}=\left(k_{n}\right)^{1 / b}\left(\frac{V_{n}}{V_{e}}\right)^{1 / b} \\
& =\left(\frac{1}{V} \int\left(\frac{\sigma}{\sigma_{\max , n}}\right)^{b} \mathrm{~d} V\right)^{1 / b}\left(\frac{V_{n}}{V_{e}}\right)^{1 / b}
\end{aligned}
$$

However, if the materials contain some pores or inclusions, Equation (23) must be used. It is important to state that Equations (23) and (24) can be used only if subsurface crack initiation is the failure process, if crack originnates from the surface, then the volume parameter in this equation should be replace with the surface area.

\section{Closed Form Solution for Fatigue Notch Factor}

To resolve inelastic deformation at the scale of microstructure to facilitate next generation microstructuresensitive notch root analyses inherently requires mesh refinement to the scale of microstructure, which is often several orders of magnitude finer than the scale of the component. Moreover, the kind of constitutive equations that must be used are often of advanced form and requiring rather sophisticated and time-consuming computational strategies to perform concurrent analyses at the component and notch root microstructure scales. Accordingly, direct application of multiscale finite element analysis is simply too computationally time consuming for practical microstructure-sensitive fatigue damage assessment of notched components under multiaxial loads. Thus, for practical engineering application, a more simplified and approximate model for fatigue notch factor is presented here based on closed form solution for stress distribution at the notch developed by Glinka using the Creager-Paris solutions of the stress field ahead of a crack. For a notched component with notch root radius $\rho$ and stress concentration factor, $k_{t}$, the axial stress distribution along the notch root centre line is given as:

$$
\begin{aligned}
\sigma & =\frac{1}{2}\left(\left(\frac{\rho}{x+\rho / 2}\right)^{1 / 2}+\frac{1}{2}\left(\frac{\rho}{x+\rho / 2}\right)^{3 / 2}\right) k_{t} S \\
& =\frac{1}{2}\left(\left(\frac{\rho}{x+\rho / 2}\right)^{1 / 2}+\frac{1}{2}\left(\frac{\rho}{x+\rho / 2}\right)^{3 / 2}\right) \sigma_{\max , n}
\end{aligned}
$$

Finding the ratio of the stress amplitude to the maximum stress and substituting into Equation (24) at $x=a_{\mathrm{c}}$ (i.e., the critical distance) will allow the determination of an expression for the fatigue notch factor of the form

$$
\begin{aligned}
k_{f}= & \left(\frac{1}{V} \int\left(\frac{1}{2}\left(\left(\frac{\rho}{a_{\mathrm{c}}+\rho / 2}\right)^{1 / 2}+\frac{1}{2}\left(\frac{\rho}{a_{\mathrm{c}}+\rho / 2}\right)^{3 / 2}\right)\right)^{b} \mathrm{~d} V\right)^{1 / b} \\
& \left(\frac{V_{n}}{V_{e}}\right)^{1 / b}
\end{aligned}
$$

Assuming that the critical distance is constant for the notched component with a notch root radius $\rho$, Equation (26) reduces to

$$
k_{f}=\left(\frac{1}{2}\left(\left(\frac{\rho}{a_{\mathrm{c}}+\rho / 2}\right)^{1 / 2}+\frac{1}{2}\left(\frac{\rho}{a_{\mathrm{c}}+\rho / 2}\right)^{3 / 2}\right)\right)\left(\frac{V_{n}}{V_{e}}\right)^{1 / b}
$$

The above equation for $k_{f}$ was derived using the fatigue damage process zone based on critical distance, 
probabilistic framework based on the weakest link, and the Glinka's closed form solution based on the notch root stress distribution. The expression in Equation (25) has been shown to give a good prediction of the stress field for relatively blunt U-notches and be used over a distance of $3 \rho$ from the notch root with an accuracy of approximately $7 \%[15]$.

\section{Results and Discussion}

The stress distribution obtained from the finite element analysis was used in determining the average $k_{f}$ for the geometry using the proposed probabilistic framework based on Weibull's weakest link and extreme-value statistics. Also as a further validation of the proposed ap- proach, the value of $k_{f}$ was calculated using the closed form solution for fatigue notch factor in Equation (27) and the result is compared to experimental results determined by R.A. Naik et al. [15] as shown in Table 2. Both results are in agreement with the experimental results with minimal difference. The $k_{f}$ determined using the Weibull's weakest link approach, when the loading ratio $\mathrm{R}=-1$, is more accurate than for every other loading ratios tested.

Also, the radius of curvature at the notch root plays a vital role on the stress gradient at the notch. This effect is captured by the fatigue notch sensitivity factor q given in Equation (3). Figure 6 gives a plot of the notch sensitiveity factor as a function of the notch root radius for the

Table 2. Comparison of measured and predicted $K_{f}$ using FEM and closed form analysis.

\begin{tabular}{|c|c|c|c|c|c|c|c|}
\hline Test Case & $K_{t}$ & $\begin{array}{l}\text { Notch radius, } \\
\rho(\mathrm{mm})\end{array}$ & $\begin{array}{l}\text { Notch depth, } \\
\text { h (mm) }\end{array}$ & R-ratio & Experimental average $K_{f}$ & $\begin{array}{c}K_{f} \text { using Weibull's weakest } \\
\text { link }\end{array}$ & $\begin{array}{c}K_{f} \text { using closed-form } \\
\text { analysis }\end{array}$ \\
\hline 1 & 2.78 & 0.330 & 0.729 & -1 & 2.79 & 2.73 & 2.66 \\
\hline 2 & 2.78 & 0.330 & 0.729 & 0.1 & 1.80 & 1.89 & 1.88 \\
\hline 3 & 2.78 & 0.330 & 0.729 & 0.5 & 1.75 & 1.82 & 1.84 \\
\hline 5 & 2.78 & 0.203 & 0.254 & 0.5 & 1.74 & 1.83 & 1.83 \\
\hline 6 & 2.78 & 0.127 & 0.127 & 0.1 & 1.98 & 2.05 & 2.01 \\
\hline 7 & 2.78 & 0.127 & 0.127 & 0.5 & 1.65 & 1.72 & 1.77 \\
\hline
\end{tabular}

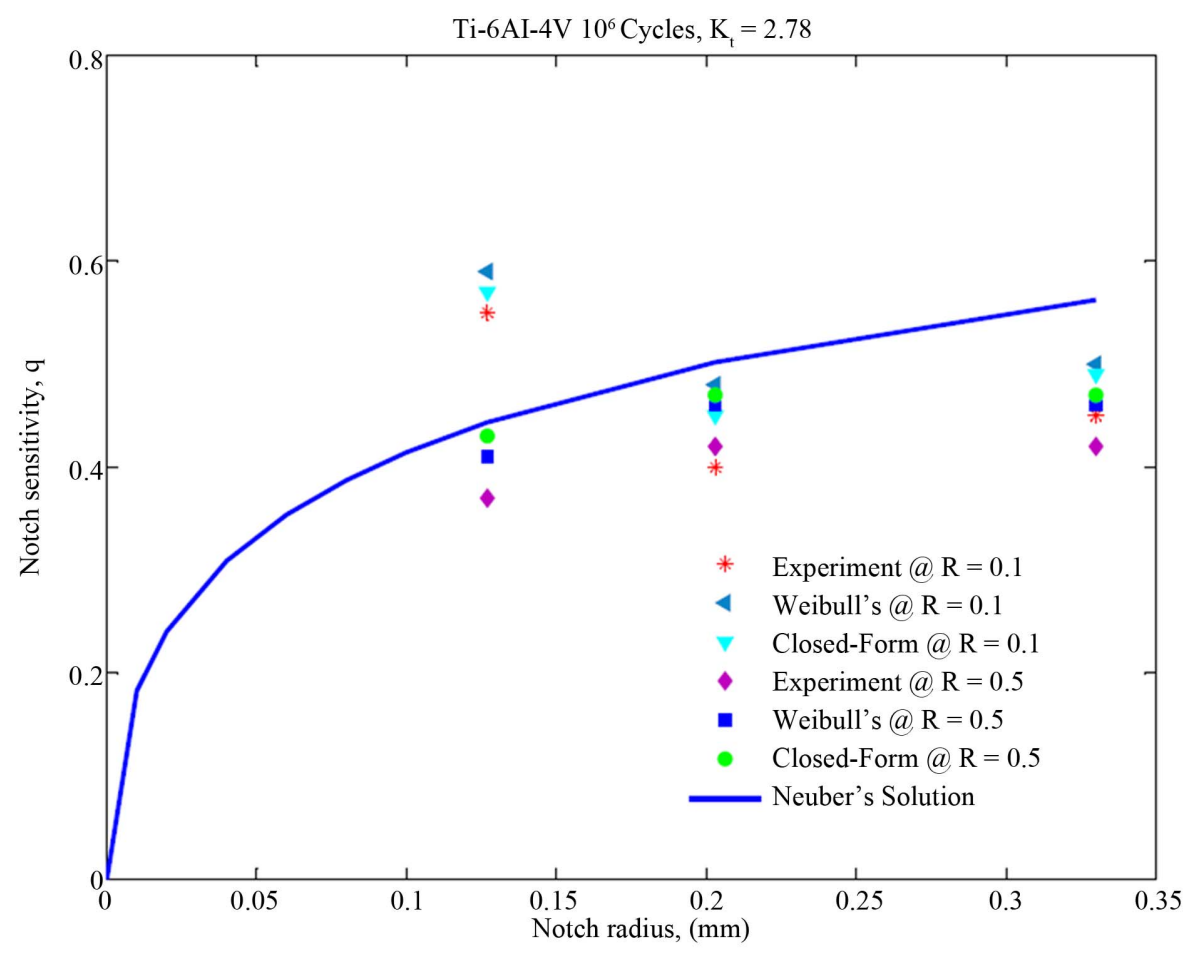

Figure 6. Notch sensitivity versus notch root radius for three notch sizes. 
different load ratio. The line plot represents the notch sensitivity factor calculated using the Neuber's formulation [7] given in Equation (28) where $a_{0}$ is a material constant taken to be 0.2 for Ti-6Al-4V [1]. The plot shows that the new approach and the closed-form solutions give more accurate result compared to the existing Neuber's formulation.

$$
q=\frac{1}{1+\sqrt{\frac{a_{0}}{\rho}}}
$$

\section{Conclusion}

A probabilistic framework based on Weibull's weakest link extreme-value statistic that accounts for stress gradient at the notch root and the realistic microstructure of the material is presented. The fatigue notch factors, $k_{f}$, estimated by this approach are compared with experimental $k_{f}$. The comparison shows that the proposed method accurately predicts the fatigue notch factor of Ti-6Al-4V for the different Load ratios applied to the specimen geometry analyzed. Also, $k_{f}$ determined from a closed form solution of the proposed probabilistic approach validates the effectiveness of the proposed method. A plot of the notch sensitivity index against the notch root radius analyzed for the different load ratios as shown in Figure 6 shows that the proposed approach gives more accurate result compared to the existing Neuber's formulation.

\section{Acknowledgements}

The authors of this paper express their profound gratitude to the Department of Defense for the financial support provided through research and educational program for HBCU/MSI (contract \#W911NF-11-1-014, L. Russel and D. Stargel, Program Managers.)

\section{REFERENCES}

[1] G. K. Haritos, T. Nicholas and D. B. Lanning, "Notch Size Effects in HCF Behavior of Ti-6Al-4V," International Journal of Fatigue, Vol. 21, No. 7, 1999, pp. 643652. doi:10.1016/S0142-1123(99)00023-7

[2] Y. Yamashita, Y. Ueda, H. Kuroki and M. Shinozaki, "Fatigue Life Prediction of Small Notched Ti-6Al-4V Specimens Using Critical Distance," Engineering Fracture Mechanics, Vol. 77, No. 9, 2010, pp. 1439-1453. doi:10.1016/j.engfracmech.2010.04.001

[3] R. E. Peterson, "Stress Concentration Factors," Wiley, New York, 1974.

[4] C. E. Philip and R. B. Heywood, "The Size Effect in Fatigue of Plain and Notched Steel Specimens under Reversed Direct Stress," Proceedings of the Institution of Mechanical Engineers, Vol. 165, 1951, pp. 113-124. doi:10.1243/PIME_PROC_1951_165_014_02
[5] D. Bellett, D. Taylor, S. Marco, E. Mazzeo, J. Guillois and T. Pircher, "The Fatigue Behaviour of Three-Dimensional Stress Concentrations," International Journal of Fatigue, Vol. 27, No. 3, 2005, pp. 207-221. doi:10.1016/j.ijfatigue.2004.07.006

[6] H. Neuber, "Theory of Stress Concentration in Shear Strained Prismatic Bodies with Arbitrary Non-Linear Stress Law," Journal of Applied Mathematics and Mechanics, Vol. 28, No. 4, 1961, pp. 544-550. doi:10.1115/1.3641780

[7] H. Neuber, "Theory of Notch Stresses: Principle for Exact Stress Calculations," Edwards, Ann Arbor, 1946.

[8] R. E. Peterson, "Notch sensitivity," In: G. Sines and J. L. Waisman, Eds., Metal Fatigue, McGraw-Hill, New York, 1959, pp. 293-306.

[9] R. B. Heywood, "Stress Concentration Factors, Relating Theoretical and Practical Factors in Fatigue Loading," Engineering, Vol. 179, 1955, pp. 146-148.

[10] Y. Weixing, "Stress Field Intensity Approach for Predicting Fatigue Life," International Journal of Fatigue, Vol. 15, No. 3, 1993, pp. 243-246. doi:10.1016/0142-1123(93)90182-P

[11] T. Anderson, "Fracture Mechanics: Fundametals and Applications," 3rd Edition, Taylor \& Francis, Boca Raton, 2005.

[12] W. D. Musinski, "Novel Methods for MicrostructureSensitive Probabilistic Fatigue Factor," Ph.D. Thesis, Georgia Institute of Technology, Atlanta, 2010.

[13] D. B. Lanning, T. Nicholas and G. K. Haritos, "On the Use of Critical Distance Theories for the Prediction of the High Cycle Fatigue Limit Stress in Notched Ti-6Al-4V," International Journal of Fatigue, Vol. 27, No. 1, 2005, pp. 45-57. doi:10.1016/j.ijfatigue.2004.06.002

[14] G. M. Owolabi, R. Prasannavenkatesan and D. L. McDowell, "Probabilistic Framework for a Microstructure-Sensitive Fatigue Notch Factor," International Journal of Fatigue, Vol. 32, No. 8, 2010, pp. 1378-1388. doi:10.1016/j.ijfatigue.2010.02.003

[15] R. A. Naik, D. B. Lanning, T. Nicholas and A. R. Kallmeyer, "A Critical Plane Gradient Approach for the Prediction of Notched HCF Life," International Journal of Fatigue, Vol. 27, No. 5, 2005, pp. 481-492. doi:10.1016/j.ijfatigue.2004.10.003

[16] R. Morrissey, C. H. Goh and D. L. McDowell, "Microstructure-Scale Modeling of HCF Deformation," Mechanics of Materials, Vol. 35, No. 3-6, 2005, pp. 295-311. doi:10.1016/S0167-6636(02)00282-X

[17] M. M. Shenoy, "Constitutive Modeling and Life Prediction in Ni-Base Superalloys," Ph.D. Thesis, Georgia Institute of Technology, 2006.

[18] R. S. Kumar, A. J. Wang and D. L. McDowell, "Effects of Microstructure Variability on Intrinsic Fatigue Resistance of Nickel-Base Superalloys-A Computational Micromechanics Approach," International Journal of Fracture, Vol. 137, No. 1-4, 2006, pp. 173-210. doi:10.1007/s10704-005-3149-y

[19] J. R. Mayeur and D. L. McDowell, "A Three-Dimensional Crystal Plasticity Model of Duplex Ti-6Al-4V," 
International Journal of Plasticity, Vol. 23, No. 9, 2007 , pp. 1457-1485. doi:10.1016/j.ijplas.2006.11.006

[20] R. J. Asaro, "Micromechanics of Crystals and Polycrystals," Advances in Applied Mechanics, Vol. 23, 1983, pp. 1-115. doi:10.1016/S0065-2156(08)70242-4

[21] R. D. McGinty, "Multiscale Representation of Polycrystalline Inelasticity," Ph.D. Thesis, Georgia Institute of Technology, Atlanta, 2001.

[22] M. Zhang, J. Zhang and D. L. McDowell, "Microstructure-
Based Crystal Plasticity Modeling of Cyclic Deformation of Ti-6Al-4V," International Journal of Plasticity, Vol. 23, No. 8, 2007, pp. 1328-1348. doi:10.1016/i.ijplas.2006.11.009

[23] F. Bridier, D. L. McDowell, P. Villechaise and J. Mendez, "Crystal Plasticity Modeling of Slip Activity in Ti-6Al4V under High Cycle Fatigue Loading," International Journal of Plasticity, Vol. 25, No. 6, 2009, pp. 1066-1082. doi:10.1016/j.ijplas.2008.08.004 